\title{
Identifying diabetes cases from administrative data: a population-based validation study
}

Lorraine L. Lipscombe ${ }^{1,2,3,4^{*}}$, Jeremiah Hwee ${ }^{3,5}$, Lauren Webster ${ }^{3}$, Baiju R. Shah ${ }^{2,3,4,6}$, Gillian L. Booth ${ }^{2,3,4,7}$ and Karen $\mathrm{Tu}^{4,8,9}$

\begin{abstract}
Background: Health care data allow for the study and surveillance of chronic diseases such as diabetes. The objective of this study was to identify and validate optimal algorithms for diabetes cases within health care administrative databases for different research purposes, populations, and data sources.

Methods: We linked health care administrative databases from Ontario, Canada to a reference standard of primary care electronic medical records (EMRs). We then identified and calculated the performance characteristics of multiple adult diabetes case definitions, using combinations of data sources and time windows.

Results: The best algorithm to identify diabetes cases was the presence at any time of one hospitalization or physician claim for diabetes AND either one prescription for an anti-diabetic medication or one physician claim with a diabetesspecific fee code [sensitivity 84.2\%, specificity 99.2\%, positive predictive value (PPV) 92.5\%]. Use of physician claims alone performed almost as well: three physician claims for diabetes within one year was highly specific (sensitivity $79.9 \%$, specificity 99.1\%, PPV 91.4\%) and one physician claim at any time was highly sensitive (sensitivity 93.6\%, specificity 91.9\%, PPV 58.5\%).

Conclusions: This study identifies validated algorithms to capture diabetes cases within health care administrative databases for a range of purposes, populations and data availability. These findings are useful to study trends and outcomes of diabetes using routinely-collected health care data.
\end{abstract}

Keywords: diabetes, validation methods, administrative databases, electronic medical record data

\section{Background}

The number of people with diabetes worldwide has quadrupled in the last three decades, with a staggering 422 million individuals now affected [1]. Population-based data on diabetes trends are becoming increasingly important to assist health care planners in managing this epidemic. Health care administrative data sources are often used to identify diabetes cases, in order to determine risk factors for diabetes, to report epidemiologic trends, to track complications and outcomes within diabetes patients, and to evaluate health service utilization

\footnotetext{
* Correspondence: Lorraine.Lipscombe@wchospital.ca

'Women's College Research Institute, Women's College Hospital, 76 Grenville

Street, Toronto, ON M5S 1B1, Canada

${ }^{2}$ Department of Medicine, University of Toronto, Suite RFE 3-805, 200

Elizabeth Street, Toronto, ON M5G 2C4, Canada

Full list of author information is available at the end of the article
}

and gaps in quality of care. As with all data that is routinely collected for other purposes, accuracy and completeness of the information may be compromised due to under-reporting or misclassification of cases. Differing case definitions and algorithms may also limit comparisons between jurisdictions.

In Canada, the Canadian Chronic Disease Surveillance System (CCDSS) identifies and monitors individuals with diabetes and other chronic conditions using a common definition [2]. The CCDSS uses routinely-collected provincial health care administrative records to identify diabetes cases, which are defined based on 1 hospitalization or 2 physician visit claims over a two-year period bearing a diagnostic code for diabetes [3]. That definition was validated in Ontario against records from primary care charts and was found to have a sensitivity of $86 \%$, a specificity of

(c) The Author(s). 2018 Open Access This article is distributed under the terms of the Creative Commons Attribution 4.0 International License (http://creativecommons.org/licenses/by/4.0/), which permits unrestricted use, distribution, and 
$97 \%$, and a positive predictive value (PPV) of $80 \%$ [4]. This algorithm has been used extensively for diabetes research to report epidemiologic trends [5, 6], quantify risk factors [7-11], evaluate outcomes [12-15], and identify health care gaps [16-18]. However, while the specificity of this definition is high, it has been shown that even modest compromises in positive predictive value increases the risk of misclassification bias [19]. This may result in sizeable errors in disease prevalence in the context of relatively uncommon conditions and large sample sizes. For instance, the 2005 Ontario Diabetes Database was estimated to have a $3 \%$ 'false positive' rate and $16 \%$ 'false negative' rate, meaning that as many as 249,840 individuals were mislabelled as having diabetes and 93,102 persons without diabetes were missed altogether [19].

One way to address this issue is to limit case definitions for diabetes to those with high PPV. Generally a PPV of $70 \%$ or greater has been considered optimal for disease algorithms using administrative or claims data [20]. However an even higher PPV (e.g. > 80\%), combined with high specificity ( $>98 \%$ ) may be preferable in large study samples to minimize the inclusion of false positive cases [19]. One rule may thus not be sufficient for all purposes, populations, and database settings. First, the need to prioritize specificity and PPV (to identify a diabetes cohort) versus sensitivity and negative predictive value, NPV (to exclude persons with pre-existing diabetes) may vary for different research objectives and purposes. Second, PPV is highly dependent on the prevalence of disease in a particular population necessitating unique algorithms based on underlying prevalence (e.g. young versus older) [20]. Third, algorithms need to be flexible to account for variations in data availability across settings (e.g. medication data, special fee codes).

In that context, the objectives of this study were to determine optimal algorithms to identify diabetes cases within health care administrative databases for different research purposes, populations, and data sources, using diabetes identified in primary care electronic medical records (EMRs) as the reference standard. We provide performance characteristics (sensitivity, specificity, positive and negative predictive value) and probabilities of having diabetes with and without each algorithm [21].

\section{Methods}

\section{Setting}

We used the Electronic Medical Record Administrative data Linked Database (EMRALD), which is a comprehensive database of EMR charts from primary care physicians who use PS Suite EMR in Ontario, Canada that is linked to administrative health care data at the individual patient level using a unique identifier [22]. Data from EMRALD held at the Institute for Clinical Evaluative Sciences were used as the reference standard to assess the performance of administrative data to capture the presence of diabetes. Data are collected annually and on a voluntarily basis from physicians. EMRALD currently contains data for over 400 physicians and over 500,000 patients in 43 clinics distributed throughout Ontario. The volunteering physicians participate by signing data sharing agreements, which allows EMRALD to collect de-identified individual patient level information without patient consent because of the prescribed entity status of ICES. EMRALD collects all of the EMR patient records of the participating physicians, which includes the cumulative patient profile (problem list, past medical history, family history, risk factors, allergies, immunizations and current treatments), laboratory test results, prescriptions, specialist consultation letters, discharge summaries and diagnostic tests for all clinical encounters. Patients and physicians are not contacted or interviewed for data collection purposes. The comprehensiveness of the data has been evaluated and all data go through data quality checks after collection and before research use [22, 23].

\section{Reference standard cohort}

EMRALD data from 296 physicians and 258,760 rostered patients were collected between April and July 2013. To be included in this study, physicians had to be using the EMR for at least 1 year. Patients were included if they were 20 years of age or older as of March 31, 2011, had an EMR for at least 1 year, and were active within their physician's practice (i.e. at least one visit within 3 years) since data collection. This ensured physicians had reasonable time to populate the EMRs with the patients' full medical history and profile. Patients were identified as having diabetes if diabetes or one of its synonyms were listed in the cumulative patient profile, or they had any of the following: haemoglobin A1c greater than 7\%, two abnormal blood glucose tests [fasting blood sugar(s) greater than or equal to $7.0 \mathrm{mmol} / \mathrm{L}$, or a random blood sugar(s) greater than or equal to $11.1 \mathrm{mmol} / \mathrm{L}$ ], or a prescription for an antidiabetic medication (insulin or an oral hypoglycemic agent) unless the record reported metformin for pre-diabetes or polycystic ovarian disease. Patients with impaired glucose tolerance, impaired fasting glucose, or gestational diabetes were excluded from the case definition. This case finding method searches the EMR's structured data using the case definition defined above, and has been previously validated using manual chart abstraction [24, 25], with a sensitivity of $90.9 \%$, specificity of $99.2 \%$, and positive predictive value of $94.9 \%$.

\section{Administrative databases}

We tested algorithms to identify diabetes within the following administrative databases. We used the discharge abstracts prepared by the Canadian Institute for Health 
Information (CIHI) to identify patients who were admitted to hospitals with a diagnosis of diabetes in any of the diagnosis fields (ICD-9 code 250; ICD-10 codes E10, E11, E13, E14) available from 1988 onwards. We used the Ontario Health Insurance Plan (OHIP) database, which captures all physician services claims, to identify claims with a diagnosis of diabetes (ICD-9 code 250) available from 1991, and with diabetes-specific fee codes that are used by Ontario primary care physicians for diabetes care (K030 since 2002; K045 since 2010, K046 since 2011, and Q040 since 2006). To capture medication data, we used the Ontario Drug Benefit database, which records prescriptions for medications covered by this plan for all Ontarians aged 65 years or older and those on social assistance available from 1991. The Registered Persons database was used to collect demographic information including age and sex. All relevant records from these data sources covered fiscal years 1991 (April 1 to March 31) to 2013. All administrative and EMR data were linked through a reproducibly scrambled unique health care identifier. Patients included in the EMRALD reference standard cohort that could not be linked to administrative databases were excluded.

\section{Administrative data algorithms}

We tested various algorithms to identify diabetes cases through combinations of records from physician claims and hospital discharge abstracts, based on the presence of a diabetes diagnosis and/or prescription claims for antihyperglycemic medications. We specifically tested the performance characteristics of the standard CCDSS algorithm, which defines a diabetes case based on 2 physician claims or 1 hospitalization in a 2 -year period bearing a diagnosis of diabetes ${ }^{4}$. Additional algorithms varied based on the number of physician service claims needed $(1,2$, or 3 claims, +/- the presence of diabetes-specific fee codes), the data sources used (hospital +/- same-day surgery records) and the time window in which case definitions needed to be met (1,2, or 3 year periods). Diabetes-specific fee codes are used by primary care providers in Ontario to submit claims for comprehensive diabetes care, which are exclusively used for diabetes patients.

\section{Analysis}

Diabetes prevalence estimates were calculated using the reference standard and the specified algorithm. Sensitivity and specificity were calculated as the proportion of diabetes cases identified by tested algorithms with and without diabetes according to the EMR reference standard. Positive predictive value (PPV), negative predictive value (NPV), and kappa were also calculated. We also estimated probabilities of having diabetes with (positive) and without (negative) meeting the case definition for each algorithm. Probabilities were calculated using Bayesian analyses, based on pre-test probabilities (prevalence) and likelihood ratios $^{21}$. Ninety-five percent confidence intervals $(95 \% \mathrm{CI})$ for proportions were calculated using binomial approximation methods. We sought algorithms that maximized sensitivity and PPV, while having the shortest time frame for case definition.

For our primary analysis, we used all available years of data to define diabetes cases within administrative databases ('ever look-back period', 1991-2013). To determine the performance of algorithms that are annually updated, as a secondary analysis we limited case definitions to data available in the most recent year before the reference standard ('1 year look-back period', 2012 to 2013). Analyses were stratified to assess potential differences by sex and by age (20-40, 41-64, and $\geq 65$ years). All analyses were performed using SAS version 9.3 (SAS Institute, Cary, NC, USA).

\section{Results}

There were 296 physicians and 258,760 rostered patients under their care. Fifteen physicians were excluded because they had not been using the EMR for at least one year, including 3248 patients under their care. Of the remaining patients, 103,331 patients were $<20$ years of age and had an electronic medical record (EMR) for at least 1 year, leaving a reference cohort of 152,177 patients. Within this cohort, 16,581 (10.9\%) patients had diabetes based on the validated definition and there were 135,596 persons without diabetes (Fig. 1). The mean age of diabetes patients was 62.9 (standard deviation, SD 13.6) years and $53.5 \%$ were male. The mean age of those without diabetes was 49.0 (SD 16.7) years and $40.8 \%$ were male.

\section{Algorithms using all available data ('ever look-back period', 1991-2013)}

For the primary analysis, we tested administrative data algorithms against the EMR reference standard for which case definitions could be met at any time (1991-2013)

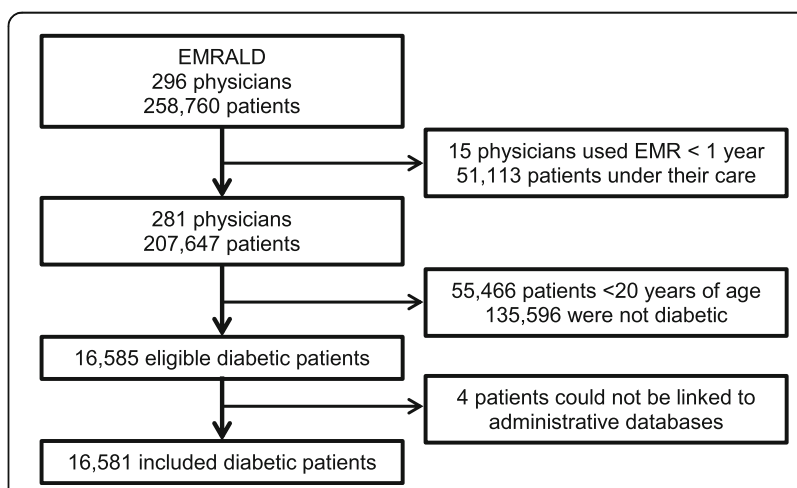

Fig. 1 Flow diagram of patients with diabetes from primary care electronic medical records 
prior to the EMR record (2012-2013). Relevant algorithms are presented in Table 1. When all available data were considered, the algorithm with the best specificity and PPV while maintaining sensitivity above $80 \%$ was either 1 hospitalization or 1 physician claim and either 1 prescription for an anti-hyperglycemic drug or 1 diabetes-specific fee code at any time (sensitivity $84.2 \%$, specificity $99.2 \%$, PPV 92.5\%). Using this algorithm, the positive probability of diabetes was $93.6 \%$ (probability that an individual meeting this definition has diabetes) and the negative probability was $2.17 \%$ (probability that an individual not meeting this definition has diabetes). Omitting prescriptions from that rule reduced sensitivity to $77.2 \%$, and omitting diabetes fee codes reduced sensitivity to $50.2 \%$ (Table 1). The algorithms with the highest sensitivity were 1 diabetes physician claim or 1 prescription at any time (94.4\%), but had moderate specificity (91.8\%) and PPV (58.4\%). The algorithm with the highest sensitivity (88.6\%) while maintaining specificity above $98 \%$ and PPV above $80 \%$ was 2 physician claims in 1 year or 1 prescription and 1 physician claim at any time (positive probability $86.1 \%$, negative probability $1.6 \%$ ).

Table 1 Validation of administrative data algorithms to adults identified with diabetes using clinical data from primary care electronic medical records as a reference standard; all adults (prevalence 10.9\%), using all administrative data available from 1991 to 2013

\begin{tabular}{|c|c|c|c|c|c|c|c|c|}
\hline Algorithm & $\begin{array}{l}\text { Sensitivity (\%) } \\
(95 \% \mathrm{Cl})\end{array}$ & $\begin{array}{l}\text { Specificity (\%) } \\
(95 \% \text { Cl) }\end{array}$ & $\begin{array}{l}\text { PPV (\%) } \\
(95 \% \text { CI) }\end{array}$ & $\begin{array}{l}\text { NPV (\%) } \\
(95 \% \text { Cl) }\end{array}$ & Prevalence & Kappa & Probability+ & Probability- \\
\hline \multicolumn{9}{|l|}{ Physician claims only } \\
\hline $1 P$ & 93.6(93.3-94.0) & $91.9(91.7-92.0)$ & $58.5(57.9-59.1)$ & 99.2(99.1-99.2) & $17.5 \%$ & 0.68 & $61.7 \%$ & $0.96 \%$ \\
\hline $2 P$ in $1 y r$ & $87.2(86.7-87.7)$ & $98.1(98.0-98.1)$ & $84.6(84.1-85.2)$ & $98.4(98.4-98.5)$ & $11.2 \%$ & 0.84 & $86.5 \%$ & $1.79 \%$ \\
\hline $3 P$ in $1 y r$ & $79.9(79.3-80.6)$ & $99.1(99.0-99.1)$ & $91.4(91.0-91.9)$ & $97.6(97.5-97.7)$ & $9.5 \%$ & 0.84 & $92.5 \%$ & $2.75 \%$ \\
\hline $2 P$ in $2 y r$ & $88.4(87.9-88.9)$ & $97.8(97.8-97.9)$ & $83.4(82.9-84.0)$ & $98.6(98.5-98.6)$ & $11.6 \%$ & 0.84 & $84.9 \%$ & $1.63 \%$ \\
\hline $3 P$ in $2 y r$ & $83.1(82.5-83.7)$ & $98.9(98.8-98.9)$ & $90.1(89.7-90.6)$ & $98.0(97.9-98.0)$ & $10.1 \%$ & 0.85 & $91.3 \%$ & $2.33 \%$ \\
\hline \multicolumn{9}{|l|}{ Inclusion of prescription claims } \\
\hline $1 \mathrm{Rx}$ & $50.7(50.0-51.5)$ & 99.8(99.8-99.9) & 97.3(97.0-97.7) & $94.3(94.2-94.4)$ & $5.7 \%$ & 0.64 & $97.2 \%$ & $6.44 \%$ \\
\hline $1 \mathrm{P}$ or $1 \mathrm{Rx}$ & $94.4(94.0-94.7)$ & 91.8(91.6-91.9) & $58.4(57.8-59.0)$ & 99.3(99.2-99.3) & $17.6 \%$ & 0.68 & $61.6 \%$ & $0.84 \%$ \\
\hline $1 \mathrm{P}$ and $1 \mathrm{Rx}$ & $50.0(49.3-50.8)$ & 99.9(99.9-99.9) & 98.5(98.3-98.8) & $94.2(94.1-94.4)$ & $5.5 \%$ & 0.64 & $98.6 \%$ & $6.52 \%$ \\
\hline$(2 \mathrm{P}$ in $1 \mathrm{yr})$ or $(1 \mathrm{Rx}$ and $1 \mathrm{P})$ & 88.6(88.1-89.1) & $98.0(98.0-98.1)$ & $84.6(84.1-85.1)$ & 98.6(98.5-98.7) & $11.4 \%$ & 0.85 & $86.1 \%$ & $1.60 \%$ \\
\hline \multicolumn{9}{|l|}{ Inclusion of hospital records } \\
\hline $\mathrm{H}$ & $36.7(36.0-37.5)$ & 99.6(99.6-99.6) & $91.9(91.2-92.5)$ & $92.8(92.7-92.9)$ & $4.4 \%$ & 0.49 & $92.7 \%$ & $8.14 \%$ \\
\hline $\mathrm{H}$ or $1 \mathrm{P}$ & $94.0(93.6-94.3)$ & $91.7(91.5-91.8)$ & $58.0(57.4-58.6)$ & $99.2(99.2-99.2)$ & $17.7 \%$ & 0.67 & $61.2 \%$ & $0.90 \%$ \\
\hline $\mathrm{H}$ or $1 \mathrm{Rx}$ & $61.3(60.5-62.0)$ & $99.5(99.4-99.5)$ & 93.3(92.8-93.7) & 95.5(95.3-95.6) & $7.2 \%$ & 0.72 & $94.5 \%$ & $5.14 \%$ \\
\hline $\mathrm{H}$ or $(2 \mathrm{P}$ in $1 \mathrm{yr})$ & 88.4(87.9-88.8) & $97.8(97.7-97.9)$ & $83.0(82.4-83.5)$ & 98.6(98.5-98.6) & $11.6 \%$ & 0.84 & $84.9 \%$ & $1.63 \%$ \\
\hline $\mathrm{H}$ or $(3 \mathrm{P}$ in $1 \mathrm{yr})$ & $82.4(81.8-83.0)$ & 98.8(98.7-98.8) & $89.1(88.6-89.6)$ & 97.9(97.8-97.9) & $10.1 \%$ & 0.84 & $90.5 \%$ & $2.42 \%$ \\
\hline $\mathrm{H}$ or $(2 \mathrm{P} \text { in } 2 \mathrm{yr})^{\mathrm{a}}$ & 89.3(88.9-89.8) & $97.6(97.5-97.7)$ & $81.9(81.3-82.4)$ & $98.7(98.6-98.7)$ & $11.9 \%$ & 0.84 & $83.8 \%$ & $1.51 \%$ \\
\hline $\mathrm{H}$ or $(3 \mathrm{P}$ in $2 \mathrm{yr})$ & $84.9(84.4-85.5)$ & $98.6(98.5-98.6)$ & $88.0(87.5-88.5)$ & $98.2(98.1-98.2)$ & $10.5 \%$ & 0.85 & $89.4 \%$ & $2.09 \%$ \\
\hline \multicolumn{9}{|c|}{ Physician claims, hospital records, and prescription claims } \\
\hline$(\mathrm{H}$ or $(2 \mathrm{P}$ in $1 \mathrm{yr}))$ or $1 \mathrm{Rx}$ & $90.0(89.5-90.4)$ & $97.7(97.6-97.8)$ & $82.6(82.0-83.1)$ & $98.8(98.7-98.8)$ & $11.9 \%$ & 0.84 & $84.5 \%$ & $1.41 \%$ \\
\hline$(\mathrm{H}$ or $(2 \mathrm{P}$ in $2 \mathrm{yr}))$ or $1 \mathrm{Rx}$ & $90.7(90.3-91.2)$ & $97.5(97.4-97.6)$ & $81.5(80.9-82.0)$ & $98.9(98.8-98.9)$ & $12.1 \%$ & 0.84 & $83.5 \%$ & $1.31 \%$ \\
\hline$(\mathrm{H}$ or $(3 \mathrm{P}$ in $2 \mathrm{yr}))$ or $1 \mathrm{Rx}$ & $87.4(86.9-87.9)$ & $98.5(98.4-98.5)$ & $87.5(87.0-88.0)$ & $98.5(98.4-98.5)$ & $10.9 \%$ & 0.86 & $89.0 \%$ & $1.75 \%$ \\
\hline$(\mathrm{H}$ or $1 \mathrm{P})$ and $1 \mathrm{~F}$ & $77.2(76.5-77.8)$ & $99.2(99.2-99.3)$ & $92.6(92.1-93.0)$ & $97.3(97.2-97.3)$ & $9.1 \%$ & 0.82 & $93.1 \%$ & $3.10 \%$ \\
\hline$(\mathrm{H}$ or $1 \mathrm{P})$ and $1 \mathrm{Rx}$ & $50.2(49.4-50.9)$ & 99.9(99.9-99.9) & 98.5(98.2-98.7) & $94.2(94.1-94.4)$ & $5.6 \%$ & 0.64 & $98.6 \%$ & $6.50 \%$ \\
\hline$(\mathrm{H}$ or $1 \mathrm{P})$ and $(1 \mathrm{Rx}$ or $1 \mathrm{~F})$ & $84.2(83.6-84.7)$ & $99.2(99.1-99.2)$ & $92.5(92.1-93.0)$ & 98.1(98.0-98.2) & $9.9 \%$ & 0.87 & $93.6 \%$ & $2.17 \%$ \\
\hline
\end{tabular}

PPV positive predictive value, NPV negative predictive value; probability+, probability of having diabetes with the algorithm, probability- probability of having disease without the algorithm, $\mathrm{H}$ hospital discharge abstracts bearing a diagnosis of diabetes from the Canadian Institute for Health Information Discharge Abstract Database, $P$ physician claims for a diabetes diagnosis (ICD-9 250) from the Ontario Health Insurance Plan Physician Claims Database, Rx prescription for an anti-hyperglycemic medication from the Ontario Drug Benefit Database, F diabetes specific physician fee code from the Ontario Health Insurance Plan Physician Claims Database, yr year

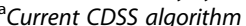

Reference standard: EMR chart - adult ( $\geq 20$ years old) with diabetes or one of its synonyms were listed in the cumulative patient profile or they had any of the following: haemoglobin A1c greater than 7\%, two abnormal blood glucose tests [fasting blood sugar(s) greater than or equal to $7.0 \mathrm{mmol} / \mathrm{L}$, or a random blood sugar(s) greater than or equal to $11.1 \mathrm{mmol} / \mathrm{L}]$, or a prescription for an anti-hyperglycemic medication (insulin or an oral hypoglycemic agent). Patients were excluded if they only had a record of gestational diabetes 
We also evaluated rules using only hospitalizations or physician claims (Table 1). If physician claims alone are used, 3 diabetes physician claims in 1 year had the best specificity (99.1\%) and PPV (91.4\%) but a sensitivity of $79.9 \%$. Using only 2 claims in 1 year increased sensitivity to $87.2 \%$ but reduced PPV to $84.6 \%$, and expanding to 3 claims over 2 years increased sensitivity to $83.1 \%$ while maintaining a high PPV (90.1\%). Of note, 2 physician claims in 1 year achieved comparable performance characteristics as the more complex algorithm identified above. One physician claim was highly sensitive (93.6\%), but had moderate specificity (91.9\%) and PPV (58.5\%). One hospitalization alone was highly specific $(99.6 \%)$ with a high PPV (91.9\%); however sensitivity was only $36.7 \%$ (negative probability $8.1 \%$ ).

Combining hospitalizations or physician claims reduced specificity and PPV in all cases, with only modest increases in sensitivity. The CCDSS algorithm of 1 hospitalization or 2 physician claims in 2 years was associated with a sensitivity of $89.3 \%$, a specificity of $97.6 \%$ and a PPV of $81.9 \%$. In contrast, 2 physician claims in 2 years alone had a higher PPV of $83.4 \%$, comparable specificity $(97.8 \%$ ) and sensitivity was only reduced to $88.4 \%$. Modifying the CCDSS algorithm to a hospitalization or 2 physician claims in 1 year also had better PPV with similar sensitivity. Adding prescription data to the CCDSS algorithm had negligible effects on performance, and adding physician claims with diabetes-specific fee codes increased sensitivity to $91.0 \%$, but reduced specificity and PPV to $97.1 \%$ and $79.1 \%$ respectively (Table 1 ).

Diabetes prevalence using the optimal algorithm was 9 . $9 \%$, compared to the reference standard of $10.9 \%$ patients with diabetes among all eligible patients in the EMR sample. In contrast, the CCDSS algorithm overestimated the prevalence at $11.9 \%$. The algorithm that most closely matched the sample prevalence was either 1 hospitalization or 3 physician claims in 2 years, or 1 prescription alone for an anti-hyperglycemic drug (10.9\%). Optimal algorithms all showed good agreement between data sources with kappa values above 0.8 (Table 1 ).

Performance characteristics of these algorithms did not vary by sex (data not shown), but varied by age group (Additional file 1 eTable S1) largely due to differences in diabetes prevalence and data availability (i.e. prescription data are available universally only for persons aged $\geq 65$ years). As the sample prevalence was only $2.13 \%$ in persons aged 20 to 40 years, the sensitivity and PPV of any given algorithm were reduced while specificity was increased. For this age group, the highest PPV (81.1\%) with reasonable sensitivity $(77.8 \%)$ was 3 physician claims in 2 years. Two physician claims in 1 year provided higher sensitivity $(81.4 \%)$ while maintaining a PPV of $70.4 \%$. In persons aged 41-64 years, 3 physician claims in 2 years was also associated with optimal sensitivity $(81.1 \%)$ and PPV (90.6\%) while 2 physician claims in 1 or 2 years increased sensitivity while maintaining a PPV above $80 \%$. In the age $65+$ group, due to the high diabetes prevalence (23.5\%) most algorithms were associated with higher PPV and sensitivity but lower specificity than other age groups. As in the overall population, the best algorithm for age $65+$ years was 1 hospitalization or physician claim and 1 prescription or diabetes-specific fee code (sensitivity 90.7\%, PPV 92.6). Using physician claims alone, 3 claims in 2 years performed best. Of note, 1 prescription for an anti-hyperglycemic drug at any time was associated with a sensitivity of $77.1 \%$, specificity of $99.5 \%$, and a PPV of 98 . $1 \%$ in persons aged 65 or older.

\section{Algorithms using most recent year of data ('1 year look-back period', 2012-2013)}

We then tested algorithms using only the most recent year of administrative data prior to the reference standard diagnosis ('1-year look-back' period, 2012-2013). This approach resulted in much lower sensitivity but higher specificity for all algorithms compared to the use of all available data (Table 2). One hospitalization or 1 physician claim for diabetes within the previous year had a sensitivity of $77.9 \%$, specificity of $99.2 \%$, and a PPV of $92.3 \%$. The best sensitivity was achieved with 1 physician claim or 1 prescription for an anti-hyperglycemic medication within the previous year, with a sensitivity of $82.6 \%$, specificity of $99.2 \%$, and PPV of 98.5\%. Therefore using a 1-year lookback period provides good positive probability but negative probability remains modest for most algorithms.

\section{Discussion}

This study used linked EMR data to provide a set of validated algorithms to identify diabetes cases within health care administrative databases. The algorithm with the best performance characteristics used linked data from hospitalization, physician claim, and prescription databases. A combination of one hospitalization or physician claim for a diabetes diagnosis AND one prescription for an anti-diabetic medication or 1 physician claim with a diabetes-specific fee code at any time was associated with a positive predictive value of $93 \%$, a specificity of $99 \%$, and a sensitivity of $84 \%$. These findings suggest that health care administrative data can accurately capture the majority of diabetes patients receiving care within the health care system. While this algorithm demonstrated good performance, it requires linkage of physician claims to prescription data and incorporates a diabetes-specific diabetes fee code only available in the province of Ontario limiting its utility to the Ontario setting. However, we found that algorithms relying on physician claims for diabetes alone performed almost as well thus making them applicable to other health care settings.

Previous validation studies identified the algorithm of 1 hospitalization or 2 physician claims in any 2 -year period 
Table 2 Validation of administrative data algorithms to identify adults identified with diabetes using clinical data from primary care electronic medical records as a reference standard; all adults, using the most recent year of administrative data (2012-2013)

\begin{tabular}{|c|c|c|c|c|c|c|c|c|}
\hline Algorithm & $\begin{array}{l}\text { Sensitivity (\%) } \\
(95 \% \text { Cl) }\end{array}$ & $\begin{array}{l}\text { Specificity (\%) } \\
(95 \% \mathrm{Cl})\end{array}$ & $\begin{array}{l}\text { PPV (\%) } \\
(95 \% \text { CI) }\end{array}$ & $\begin{array}{l}\text { NPV (\%) } \\
(95 \% \text { Cl) }\end{array}$ & Prevalence & Kappa & Probability+ & Probability \\
\hline \multicolumn{9}{|l|}{ Physician claims only } \\
\hline $1 \mathrm{P}$ & 76.4(75.8-77.1) & 99.2(99.2-99.3) & 92.3(91.9-92.7) & $97.2(97.1-97.3)$ & $9.01 \%$ & 0.82 & $92.1 \%$ & $3.21 \%$ \\
\hline $2 \mathrm{P}$ in $1 \mathrm{yr}$ & $58.5(57.8-59.3)$ & 99.8(99.8-99.8) & $97.4(97.1-97.7)$ & $95.2(95.1-95.3)$ & $6.54 \%$ & 0.71 & $97.3 \%$ & $5.48 \%$ \\
\hline $3 \mathrm{P}$ in $1 \mathrm{yr}$ & $41.2(40.5-42.0)$ & 99.9(99.9-99.9) & 98.3(98.0-98.6) & 93.3(93.2-93.4) & $4.57 \%$ & 0.55 & $98.1 \%$ & $7.58 \%$ \\
\hline \multicolumn{9}{|l|}{ Inclusion of prescription claims } \\
\hline $1 \mathrm{Rx}$ & $45.9(45.2-46.7)$ & 100.0(100.0-100.0) & 99.3(99.1-99.5) & 93.8(93.7-93.9) & $5.03 \%$ & 0.60 & - & $7.01 \%$ \\
\hline $1 \mathrm{P}$ or $1 \mathrm{Rx}$ & $82.6(82.1-83.2)$ & $99.2(99.1-99.2)$ & $92.6(92.2-93.0)$ & $97.9(97.8-98.0)$ & $9.72 \%$ & 0.86 & $92.7 \%$ & $2.39 \%$ \\
\hline $1 \mathrm{P}$ and $1 \mathrm{Rx}$ & $39.7(39.0-40.5)$ & 100.0(100.0-100.0) & 99.8(99.7-99.9) & $93.1(93.0-93.3)$ & $4.33 \%$ & 0.54 & - & $7.75 \%$ \\
\hline$(2 \mathrm{P}$ in $1 \mathrm{yr}$ ) or (1Rx and $1 \mathrm{P})$ & $65.9(65.1-66.6)$ & 99.8(99.8-99.8) & 97.6(97.4-97.9) & 96.0(95.9-96.1) & $7.35 \%$ & 0.77 & $97.6 \%$ & $4.55 \%$ \\
\hline \multicolumn{9}{|l|}{ Inclusion of hospital records } \\
\hline $\mathrm{H}$ & $9.8(9.4-10.3)$ & 99.9(99.9-100.0) & 95.6(94.6-96.6) & $90.1(89.9-90.2)$ & $1.12 \%$ & 0.16 & $92.3 \%$ & $11.18 \%$ \\
\hline $\mathrm{H}$ or $1 \mathrm{P}$ & $77.9(77.2-78.5)$ & $99.2(99.1-99.2)$ & $92.0(91.6-92.5)$ & 97.3(97.3-97.4) & $9.21 \%$ & 0.83 & $92.3 \%$ & $3.01 \%$ \\
\hline $\mathrm{H}$ or $1 \mathrm{Rx}$ & 48.6(47.9-49.4) & 99.9(99.9-99.9) & $98.5(98.2-98.8)$ & $94.1(94.0-94.2)$ & $5.38 \%$ & 0.62 & $98.3 \%$ & $6.69 \%$ \\
\hline $\mathrm{H}$ or (2 $\mathrm{P}$ in $1 \mathrm{yr})$ & $61.6(60.9-62.4)$ & 99.8(99.7-99.8) & $96.9(96.6-97.3)$ & 95.5(95.4-95.6) & $6.92 \%$ & 0.73 & $97.4 \%$ & $5.09 \%$ \\
\hline $\mathrm{H}$ or (3 $\mathrm{P}$ in $1 \mathrm{yr})$ & $45.7(44.9-46.4)$ & 99.9(99.8-99.9) & 97.6(97.2-97.9) & 93.8(93.6-93.9) & $5.10 \%$ & 0.59 & $98.2 \%$ & $7.04 \%$ \\
\hline \multicolumn{9}{|c|}{ Physician claims, hospital records, prescription claims } \\
\hline$(\mathrm{H}$ or $(2 \mathrm{P}$ in $1 \mathrm{yr}))$ or $1 \mathrm{Rx}$ & $73.1(72.5-73.8)$ & 99.7(99.7-99.8) & $97.1(96.8-97.4)$ & $96.8(96.7-96.9)$ & $8.21 \%$ & 0.82 & $96.8 \%$ & $3.62 \%$ \\
\hline$(\mathrm{H}$ or $1 \mathrm{P})$ and $(1 \mathrm{Rx}$ or $1 \mathrm{~F})$ & $67.3(66.6-68.0)$ & 99.8(99.7-99.8) & $97.3(97.0-97.6)$ & $96.1(96.0-96.2)$ & $7.53 \%$ & 0.78 & $97.6 \%$ & $4.37 \%$ \\
\hline$(\mathrm{H}$ or $1 \mathrm{P})$ and $1 \mathrm{~F}$ & $56.4(55.7-57.2)$ & 99.8(99.8-99.8) & $96.9(96.5-97.2)$ & $94.9(94.8-95.0)$ & $6.34 \%$ & 0.69 & $97.2 \%$ & $5.74 \%$ \\
\hline$(\mathrm{H}$ or $1 \mathrm{P})$ and $1 \mathrm{Rx}$ & 40.6(39.9-41.4) & $100.0(100.0-100.0)$ & 99.8(99.7-99.9) & 93.2(93.1-93.4) & $4.43 \%$ & 0.55 & - & $7.65 \%$ \\
\hline
\end{tabular}

PPV positive predictive value, NPV negative predictive value, $H$ hospital discharge abstracts bearing a diagnosis of diabetes from the Canadian Institute for Health Information Discharge Abstract Database, $P$ physician claims for a diabetes diagnosis (ICD-92 50) from the Ontario Health Insurance Plan Physician Claims Database, $R x$ prescription for an anti-hyperglycemic medication from the Ontario Drug Benefit Database, $F$, diabetes specific physician feecode from the Ontario Health Insurance Plan Physician Claims Database, $S$ same day surgery admission bearing a diagnosis of diabetes from the Canadian Institute for Health Information Discharge Abstract Database; yr., year

Reference standard: EMR chart - adult ( $\geq 20$ years old) with diabetes or one of its synonyms were listed in the cumulative patient profile or they had any of the following: haemoglobin A1c greater than 7\%, two abnormal blood glucose tests [fasting blood sugar(s) greater than or equal to $7.0 \mathrm{mmol} / \mathrm{L}$, or a random blood sugar(s) greater than or equal to $11.1 \mathrm{mmol} / \mathrm{L}$, or a prescription for an anti-hyperglycemic medication (insulin or an oral hypoglycemic agent). Patients were excluded if they only had a record of gestational diabetes

as having the best performance characteristics [3, 4]. This algorithm has been widely used by the CCDSS and Canadian researchers to study the trends, care, and outcomes of diabetes. Our study expanded on those findings to test a larger number of algorithms. Reassuringly, we confirmed that this algorithm continues to perform well: it was associated with $89 \%$ sensitivity, $98 \%$ specificity, and a positive predictive value of $82 \%$. However, we found that the inclusion of hospitalization data only increases sensitivity by $1 \%$ and leads to a lower positive predictive value compared to physician claims alone. Simpler algorithms that used only physician claims data had comparable or better positive predictive value as the more complex CCDSS algorithm. The highest positive predictive value (91\%) and specificity (99\%) was achieved with 3 claims in any 2-year period, which had a sensitivity of $83 \%$. Similarly, we found that limiting the observation period to 2 physician claims in any one-year period only drops sensitivity by $2 \%$ (87\%) with maintenance of high specificity (98\%) and positive predictive value (85\%).. Our study therefore supports the use of more simplified algorithms over the more complex CCDSS algorithm, allowing for diabetes to be accurately identified using a single database and in a shorter time period. While all provinces in Canada have access to hospital discharge data from the Canadian Institute for Health Information (CIHI), the linkage of physician claims data to CIHI required by the CCDSS algorithm may be more limited in some provinces. The algorithms that use physician claims alone could therefore be widely applied across Canadian provinces. For studies requiring high specificity, using 2 (sensitivity $87 \%$, specificity $98 \%$ ) or 3 (sensitivity $80 \%$, specificity $99 \%$ ) physician claims for diabetes in a one-year period would perform best. Conversely for studies requiring high sensitivity (e.g. to exclude diabetes cases), diabetes could be defined using 1 physician claim for diabetes at any time (sensitivity $94 \%$, specificity $92 \%$ ).

The performance of algorithms varied by age group, largely due to differences in prevalence of diabetes. In 
general, a given algorithm had lower sensitivity and positive predictive value but higher specificity in younger versus older age groups, due to their lower prevalence of diabetes. Age-specific performance characteristics therefore need to be taken into consideration in diabetes studies that restrict or stratify cohorts by age group. The optimal algorithm for individuals aged 20 to 40 years was 3 physician claims in 2 years, which is comparable to the validated algorithm for pediatric cases of diabetes (4 physician claims in 2 years) [26]. Of note, because prescription medications are captured for all individuals aged 65 years or older in Ontario databases, use of any prescription record for an anti-diabetes medication alone was associated with a positive predictive value of $98 \%$ and specificity of $99.5 \%$ in that age group. These findings provide support for use of prescription data alone to identify patients with diabetes in databases where all medications are captured, such as in the Canadian provinces of British Columbia, Quebec, Saskatchewan, and Manitoba. However, as sensitivity was only $77 \%$, use of prescription claims should be limited to conditions whereby specificity is more important than sensitivity.

Previous studies did not clarify the optimal length of look-back period needed to capture or exclude baseline cases of diabetes in health care databases. We showed that a one-year look-back period is sufficient to accurately capture diabetes cases with a high positive predictive value, but sensitivity is reduced leading to a greater proportion of missed cases. Use of 1 physician claim or 1 anti-diabetic prescription in the previous year, however, was able to identify $83 \%$ of cases with a positive predictive value of almost 99\%. The advantage of having a one year look-back period allows for reporting up to the most recent year available data. Requiring a longer look-back period to meet a case definition necessitates a wider time-frame prior to cohort entry, which may compromise the eligibility of study participants and follow-up time. Although we lost sensitivity with a one year look back period, the substantial increase in positive predictive value to near optimal levels suggests a low number of false positives. Therefore the annual application of the algorithm would be an accurate and conservative approach that could help reduce the accumulation of false positives.

Strengths of this study include the use of a large population-based sample, completeness of data capture in a single-payer health care system, use of a manually validated reference standard, and testing of a large number of algorithms. We also had access to EMRs linked to administrative data, which allowed for efficient testing of multiple algorithms that would not be feasible with manual chart review [27]. However there are limitations to this study. First, our reference population was restricted to patients who had a primary care physician and had at least 1 visit within the previous 3 years. The superior performance of physician claim-based algorithms may be partly attributed to the dependence on primary care visits to identify our reference cases; we were not able to determine how well these algorithms would identify patients not actively managed by a primary care physician. This reference standard does not capture diabetes cases uniquely identified in a hospital setting; therefore our study may have underestimated the potential increase in sensitivity achieved with adding hospitalization data to physician claims. However given the nature of the disease, it is unlikely that patients with diabetes would not see their family physician at least once in a 3 -year period. We also did not have data on undiagnosed diabetes or on persons who do not regularly access the health care system. Second, as we did not have data on laboratory tests in our administrative data, we could not test algorithms that incorporate blood tests such as glucose or HbAlc. Third, while we found that prescription data alone performed well in persons aged 65 years or older, we could not determine whether this would be generalizable to younger age groups if medication data were available. Finally, our study was limited to the Ontario health care context and results may not be applicable to other settings.

\section{Conclusion}

In conclusion, we identified performance characteristics for a set of algorithms that can be used to accurately capture diabetes cases within health care administrative databases. We provide optimal algorithms overall and by age group, and using both linked and unlinked data from different databases. These findings will be useful for researchers and policymakers seeking to study trends and outcomes of diabetes within a Canadian context, and may also be applicable to other settings with similar data.

\section{Additional file}

Additional file 1: Validation of administrative data algorithms to identify adult patients who were identified with diabetes using clinical data from primary care electronic medical records as a reference standard by age group, using all administrative data available from 1991-2013. (DOCX 22 kb)

\section{Abbreviations}

CCDDS: Canadian Chronic Disease Surveillance System; CIHI: Canadian Institute for Health Information; EMR: Electronic Medical Records; EMRALD: Electronic Medical Record Administrative data Linked Database; NPV: Negative Predictive Value; OHIP: Ontario Health Insurance Plan; PPV: Positive Predictive Value

\section{Acknowledgements}

The authors would like to thank Ping Li, Diane Green, Jacqueline Young, Bogdan Pinzaru, Karen Cauch-Dudek, and Christina Yu for assistance with data extraction and analysis, administrative support, and manuscript preparation.

\section{Funding}

This study was supported by the University of Toronto Banting and Best Diabetes Centre, and by the Institute for Clinical Evaluative Sciences (ICES), which is funded by an annual grant from the Ontario Ministry of Health and 
Long-Term Care (MOHLTC). Dr. Lorraine Lipscombe was supported by a New Investigator Award from the Canadian Institutes for Health Research. Dr. Karen Tu is supported by a research scholar award from the Department of Family and Community Medicine, University of Toronto. The opinions, results and conclusions reported in this paper are those of the authors and are independent from the funding sources. No endorsement by ICES or the MOHLTC is intended or should be inferred.

\section{Availability of data and materials}

The dataset from this study is held securely in coded form at the Institute for Clinical Evaluative Sciences (ICES). While data sharing agreements prohibit ICES from making the dataset publicly available, access may be granted to those who meet pre-specified criteria for confidential access, available at https://www.ices.on.ca/DAS. The full dataset creation plan and underlying analytic code are available from the authors upon request, understanding that the programs may rely upon coding templates or macros that are unique to ICES.

\section{Authors' contributions}

LLL co-conceptualized the study and co-drafted the manuscript. JH prepared the study protocol and co-drafted the manuscript. LW assisted with data analysis and presentation of results, and edited the manuscript. BRS contributed to study design and interpretation of results, and edited the manuscript. GLB contributed to study design and interpretation of results, and edited the manuscript. KT co-conceptualized the study, contributed to the study protocol and interpretation of results, and edited the manuscript. All authors read and approved the final manuscript.

\section{Ethics approval and consent to participate}

The study protocol was approved by the Research Ethics Board at Sunnybrook Health Sciences Center.

\section{Competing interests}

The authors declare that they have no competing interests.

\section{Publisher's Note}

Springer Nature remains neutral with regard to jurisdictional claims in published maps and institutional affiliations.

\begin{abstract}
Author details
'Women's College Research Institute, Women's College Hospital, 76 Grenville Street, Toronto, ON M5S 1B1, Canada. 'Department of Medicine, University of Toronto, Suite RFE 3-805, 200 Elizabeth Street, Toronto, ON M5G 2C4, Canada. ${ }^{3}$ Institute for Clinical Evaluative Sciences, G1 06, 2075 Bayview Avenue, Toronto, ON M4N 3M5, Canada. Institute of Health Policy, Management and Evaluation, University of Toronto, 4th Floor, 155 College St, Toronto, ON M5T 3M6, Canada. ${ }^{5}$ Dalla Lana School of Public Health, University of Toronto, 6th Floor, 155 College St, Toronto, ON M5T 3M7, Canada. 'S ${ }^{6}$ unnybrook Health Sciences Centre, 2075 Bayview Avenue, Toronto, ON M4N 3M5, Canada. ${ }^{7} \mathrm{Li}$ Ka Shing Knowledge Institute, St. Michael's Hospital, 30 Bond St, Toronto, ON M5B 1W8, Canada. ${ }^{8}$ Department of Community and Family Medicine, University of Toronto, 5th Floor, 500 University Avenue, Toronto, ON M5G 1V7, Canada. ${ }^{9}$ University Health Network, R. Fraser Elliot Building, 1st Floor, 190 Elizabeth St, Toronto, ON M5G 2C4, Canada.
\end{abstract}

Received: 4 January 2018 Accepted: 25 April 2018

Published online: 02 May 2018

\section{References}

1. Collaboration NCDRF. Worldwide trends in diabetes since 1980: a pooled analysis of 751 population-based studies with 4.4 million participants. Lancet. 2016;387(10027):1513-30.

2. Clottey C, Mo F, LeBrun B, Mickelson P, Niles J, Robbins G. The development of the National Diabetes Surveillance System (NDSS) in Canada. Chronic Dis Can. 2001;22(2):67-9.

3. Blanchard JF, Ludwig S, Wajda A, et al. Incidence and prevalence of diabetes in Manitoba, 1986-1991. Diabetes Care. 1996;19(8):807-11.

4. Hux JE, Ivis F, Flintoft V, Bica A. Diabetes in Ontario: Determination of prevalence and incidence using a validated administrative data algorithm. Diabetes Care. 2002;25(3):512-6.
5. Lipscombe LL, Hux JE. Trends in diabetes prevalence, incidence, and mortality in Ontario, Canada 1995-2005: a population-based study. Lancet. 2007;369(9563):750-6.

6. Feig DS, Hwee J, Shah BR, Booth GL, Bierman AS, Lipscombe LL. Trends in incidence of diabetes in pregnancy and serious perinatal outcomes: a large, population-based study in ontario, Canada, 1996-2010. Diabetes Care. 2014; 37(6):1590-6.

7. Feig DS, Shah BR, Lipscombe $L L$, et al. Preeclampsia as a risk factor for diabetes: a population-based cohort study. PLoS Med. 2013;10(4):e1001425.

8. Feig DS, Zinman B, Wang X, Hux JE. Risk of development of diabetes mellitus after diagnosis of gestational diabetes. CMAJ. 2008;179(3):229-34

9. Booth GL, Creatore MI, Moineddin R, et al. Unwalkable neighborhoods, poverty, and the risk of diabetes among recent immigrants to Canada compared with long-term residents. Diabetes Care. 2013;36(2):302-8.

10. Lysy Z, Booth GL, Shah BR, Austin PC, Luo J, Lipscombe LL. The impact of income on the incidence of diabetes: a population-based study. Diabetes Res Clin Pract. 2013;99(3):372-9.

11. Lipscombe LL, Chan WW, Yun L, Austin PC, Anderson GM, Rochon PA. Incidence of diabetes among postmenopausal breast cancer survivors. Diabetologia. 2013;56(3):476-83.

12. Booth GL, Kapral MK, Fung K, Tu JV. Relation between age and cardiovascular disease in men and women with diabetes compared with non-diabetic people: a population-based retrospective cohort study. Lancet. 2006;368(9529):29-36.

13. Booth GL, Kapral MK, Fung K, Tu JV. Recent trends in cardiovascular complications among men and women with and without diabetes. Diabetes Care. 2006:29(1):32-7.

14. Booth GL, Hux JE. Relationship between avoidable hospitalizations for diabetes mellitus and income level. Arch Intern Med. 2003;163(1):101-6.

15. Lind $M$, Garcia-Rodriguez LA, Booth $G L$, et al. Mortality trends in patients with and without diabetes in Ontario, Canada and the UK from 1996 to 2009: a population-based study. Diabetologia. 2013;56(12):2601-8.

16. Lipscombe LL, Hux JE, Booth GL. Reduced screening mammography among women with diabetes. Arch Intern Med. 2005;165(18):2090-5.

17. Shah BR, Hux JE, Laupacis A, Zinman B. van WC. Clinical inertia in response to inadequate glycemic control: do specialists differ from primary care physicians? Diabetes Care. 2005;28(3):600-6.

18. Cheung A, Stukel TA, Alter DA, et al. Primary Care Physician Volume and Quality of Diabetes Care: A Population-Based Cohort Study. Ann Intern Med. 2017;166(4):240-7.

19. Manuel DG, Rosella LC, Stukel TA. Importance of accurately identifying disease in studies using electronic health records. BMJ. 2010;341:c4226.

20. Carnahan RM. Mini-Sentinel's systematic reviews of validated methods for identifying health outcomes using administrative data: summary of findings and suggestions for future research. Pharmacoepidemiol Drug Saf. 2012; 21(Suppl 1):90-9.

21. van Walraven C, Bennett C, Forster AJ. Administrative database research infrequently used validated diagnostic or procedural codes. J Clin Epidemiol. 2011:64(10):1054-9.

22. Tu K, Mitiku TF, Ivers NM, et al. Evaluation of Electronic Medical Record Administrative data Linked Database (EMRALD). Am J Manag Care. 2014; 20(1):e15-21.

23. Tu K, Widdifield J, Young J, et al. Are family physicians comprehensively using electronic medical records such that the data can be used for secondary purposes?A Canadian perspective. BMC Med Inform Decis Mak. 2015;15:67.

24. Tu K, Manuel D, Lam K, Kavanagh D, Mitiku TF, Guo H. Diabetics can be identified in an electronic medical record using laboratory tests and prescriptions. J Clin Epidemiol. 2011;64(4):431-5.

25. Ivers NM, Tu K, Francis J, et al. Feedback GAP: study protocol for a clusterrandomized trial of goal setting and action plans to increase the effectiveness of audit and feedback interventions in primary care. Implement Sci. 2010;5:98.

26. Guttmann A, Nakhla M, Henderson M, et al. Validation of a health administrative data algorithm for assessing the epidemiology of diabetes in Canadian children. Pediatr Diabetes. 2010;11(2):122-8.

27. Lee WJ, Lee TA, Pickard AS, Shoaibi A, Schumock GT. Using linked electronic data to validate algorithms for health outcomes in administrative databases. J Comp Eff Res. 2015;4(4):359-66. 